\title{
Isolation and characterization of polymorphic microsatellite loci in the ridgetail white prawn Exopalaemon carinicauda
}

\author{
S.W. Jia ${ }^{1,2}$, P. Liu ${ }^{1}$, J. Li ${ }^{1}$, J.T. Li ${ }^{1}$ and L.Q. Pan $^{2}$ \\ ${ }^{1}$ Key Laboratory of Sustainable Development of Marine Fisheries, \\ Ministry of Agriculture Yellow Sea Fisheries Research Institute, \\ Chinese Academy of Fishery Sciences, Qingdao, Shandong, China \\ ${ }^{2}$ Fisheries College, Ocean University of China, Qingdao, Shandong, China \\ Corresponding author: P. Liu \\ E-mail: liuping@ysfri.ac.cn
}

Genet. Mol. Res. 12 (3): 2816-2820 (2013)

Received January 10, 2012

Accepted November 22, 2012

Published August 8, 2013

DOI http://dx.doi.org/10.4238/2013.August.8.1

\begin{abstract}
The ridgetail white prawn Exopalaemon carinicauda is one of the major economic shrimp species cultured in China. In this study, 30 microsatellite loci were isolated and characterized for the ridgetail white prawn E. carinicauda using a microsatelliteenriched library. Polymorphisms were tested in 30 individuals from a single wild population. The number of alleles at each locus ranged from 2 to 14 . The observed and expected heterozygosities varied from 0.1000 to 0.8000 and from 0.2299 to 0.9228 , respectively. The PIC value ranged from 0.2002 to 0.8939 . These new loci will be useful in the study of population genetic structure and genetic diversity in this species.
\end{abstract}

Key words: Exopalaemon carinicauda; Enriched library; Microsatellites 


\section{INTRODUCTION}

The ridgetail white prawn Exopalaemon carinicauda (Crustacea: Decapoda: Palaemonidae) is an endemic shrimp species in China and is widely distributed in the coastal waters of China and west coast of the Korean Peninsula, especially in the Yellow and Bohai Seas. Due to the multiple merits of good reproductive performance, fast growth, and good environmental adaptability, the culture area of the ridgetail white prawn in China has expanded in recent years (Wang and Cao, 2010). Although the ridgetail white prawn is one of the major commercial shrimp species cultured in China and contributes one-third of the gross revenues from the polyculture ponds in eastern China (Xu et al., 2010), its seeding is dependent on wild resources.

So far, studies have focused on culture (Shi and Ye, 2007), biology (Li, 1994), and ecology (Wang and Cao, 2010), but there are few reports about population genetics. The conservation and culture of the prawns require information about the genetic diversity and structure of E. carinicauda. To provide some available information on developing conservation and culture, efficient molecular markers are needed to study the genetic diversity and structure of the species. Microsatellite markers are regarded as a useful tool for such studies, because they show a high degree of polymorphism, co-dominance and neutrality. In this study, we developed 30 polymorphic microsatellite loci in the ridgetail white prawn.

\section{MATERIAL AND METHODS}

Samples of the ridgetail white prawn were collected from the coastal waters of the Bohai Sea, Laizhou Bay, Shandong Province, China. The samples were stored at $-80^{\circ} \mathrm{C}$ in the laboratory.

The isolation of microsatellites from the prawn was performed using the enrichment protocols reported by Song (2010), with minor modifications. Genomic DNA was extracted from the muscle of a single individual using phenol-chloroform procedures described by Liu et al. (2000). Approximately $6 \mu \mathrm{g}$ genomic DNA was digested with the restriction endonuclease HaeIII. DNA fragments from 400 to $1200 \mathrm{bp}$ were separated on a $1.5 \%$ agarose gel, extracted using a Gel Extraction kit (Tiangen, China), and ligated to HaeIII adaptors generated by annealing together oligo A (5'-CTCTTGCTTGAATTCGGACTA-3') and oligo B (5'-pTAGTCCGAATTCAAGCAAGAGCACA-3') using T 4 DNA ligase (TaKaRa, Dalian, China). The ligated DNA fragments were amplified by polymerase chain reaction (PCR) using oligo A as the primer. The PCR products were cleaned using a DNA purification kit. The PCR products were then hybridized with the biotinylated probes $(\mathrm{AC})_{15}$ and $(\mathrm{AG})_{15}$, and the hybridization complexes were captured using streptavidin magnetic beads (Promega). Captured fragments were amplified again using oligo A as primer, ligated to pMD18-T vector (TaKaRa), and transformed into competent Escherichia coli DH5 $\alpha$ cells. The clones were identified on LB plus ampicillin plates. Approximately 460 clones were randomly picked and screened by colony PCR and 314 clones were positive. A total of 227 positive colonies were selected from the 314 clones and sequenced on an ABI 3730 DNA sequencer (Applied Biosystems), which showed that 186 clones contained microsatellites.

After we discarded duplicates using the DNAMAN version 6.0 software and discarded those with short unique regions flanking the microsatellite array, 132 primer pairs were designed by the Primer 5.0 software (http://www.premierbiosoft.com/). Primers were selected 
and PCR conditions were optimized using genomic DNA obtained from 5 individuals. The polymorphisms of the microsatellite loci were examined in 30 individuals of E. carinicauda. The PCR amplifications were done in a $20-\mu \mathrm{L}$ reaction volume containing $90 \mathrm{ng}$ DNA template, $1.0 \mu \mathrm{M}$ forward and reverse primers, 1X PCR buffer, $2.4 \mathrm{mM} \mathrm{MgCl}_{2}, 0.32 \mathrm{mM}$ dNTPs, and 1.0 U Taq DNA polymerase. PCR profiles involved an initial denaturation at $94^{\circ} \mathrm{C}$ for $5 \mathrm{~min}$, followed by 28 cycles of $94^{\circ} \mathrm{C}$ for $45 \mathrm{~s}$, annealing at $48^{\circ}$ to $62^{\circ} \mathrm{C}$ (Table 1) for $45 \mathrm{~s}$ and extension at $72^{\circ} \mathrm{C}$ for $45 \mathrm{~s}$, and a final extension at $72^{\circ} \mathrm{C}$ for $5 \mathrm{~min}$.

PCR products were resolved on $8 \%$ denaturing polyacrylamide gels and visualized by silver staining. We calculated the number of alleles per locus. The observed and the expected heterozygosities, and test for Hardy-Weinberg equilibrium (HWE) were determined with POPGENE32 (Yeh et al., 1999). Linkage disequilibrium was tested using GENEPOP 4.0 (Rousset, 2008). Polymorphism information content (PIC) was calculated using the method described by Botstein et al. (1980).

\section{RESULTS AND DISCUSSION}

Of a total of 132 microsatellite loci, 48 were polymorphic in E. carinicauda. Thirteen markers were judged to be multilocus markers because they amplified more than two alleles in most individuals. Five markers could not be fully amplified in 30 individuals of $E$. carinicauda. The number of alleles at the remaining 30 SSR markers ranged from 2 to 14 with an average of 8.2 alleles. The observed and the expected heterozygosities ranged from 0.1000 to 0.8000 and from 0.2299 to 0.9228 , with an average of 0.4438 and 0.8220 , respectively. The PIC value ranged from 0.2002 to 0.8939 (Table 1). Botstein et al. (1980) regarded any locus with a PIC $\geq$ 0.5 as highly polymorphic. In our study, only 1 of the 30 loci analyzed did not meet this criterion. Sixteen of the 30 loci deviated significantly from HWE after a Bonferroni's correction (P $<0.0017$ ). These deviations might have been caused by the presence of null alleles (Pemberton et al., 1995) and sampling effect. Significant linkage disequilibrium was not detected between any pairs of loci $(\mathrm{P}<0.01)$. The results suggested that these polymorphic loci should be useful in surveying the population genetic structure and genetic diversity of E. carinicauda.

\begin{tabular}{|c|c|c|c|c|c|c|c|c|c|c|}
\hline Locus & $\begin{array}{l}\text { Accession } \\
\text { No. }\end{array}$ & Repeat motif & Primer sequence $\left(5^{\prime}-3^{\prime}\right)$ & $\begin{array}{l}\mathrm{Ta} \\
\left({ }^{\circ} \mathrm{C}\right)\end{array}$ & $N_{\mathrm{A}}$ & $\begin{array}{l}\text { Size range } \\
\text { (bp) }\end{array}$ & $H_{\mathrm{o}}$ & $H_{\mathrm{E}}$ & $P$ & PIC \\
\hline EC65 & JQ319400 & $(\mathrm{CT})_{28} \mathrm{CC}(\mathrm{CT})_{6}$ & $\begin{array}{l}\text { CCTGAGTAACGGTCCATA } \\
\text { TACACCTCGCTCCTAAAA }\end{array}$ & 58 & 5 & $507-532$ & 0.2759 & 0.7338 & $0.0000^{*}$ & 0.6692 \\
\hline EC1125 & JN408494 & $(\mathrm{TC})_{33}$ & $\begin{array}{l}\text { TCAGCCTGAGGGTTTTGT } \\
\text { GTAGTCGCCGCAGAAGTT }\end{array}$ & 54 & 6 & $502-543$ & 0.4333 & 0.7401 & 0.0018 & 0.6830 \\
\hline EC909 & JQ319401 & $(\mathrm{TC})_{11} \mathrm{C}(\mathrm{CT})_{14}$ & $\begin{array}{l}\text { GAACCCTTTACCTACACGACTG } \\
\text { GATTGTGTTTGAGTGGAGCCCT }\end{array}$ & 48 & 6 & $416-439$ & 0.5862 & 0.7949 & 0.0336 & 0.7485 \\
\hline EC7 & JQ319402 & $(\mathrm{GA})_{29}$ & $\begin{array}{l}\text { AATATGCAGTGGCAAGCT } \\
\text { TTCCCATCATCTTCCTCC }\end{array}$ & 56 & 7 & 503-559 & 0.4000 & 0.8114 & $0.0000^{*}$ & 0.7647 \\
\hline EC54 & JQ319403 & $(\mathrm{GA})_{36}$ & $\begin{array}{l}\text { GGCTGTCCCTTGGAACTA } \\
\text { ACGAAATCCGAATAACCC }\end{array}$ & 56 & 11 & $328-367$ & 0.7000 & 0.8898 & 0.1137 & 0.8621 \\
\hline EC915 & JN408481 & $(\mathrm{AG})_{34}$ & $\begin{array}{l}\text { AAAGTGCGTTACAGGAAG } \\
\text { GTCTGGAAACACCGAATG }\end{array}$ & 51 & 9 & $358-427$ & 0.5600 & 0.8465 & $0.0000^{*}$ & 0.8086 \\
\hline EC13 & JQ319404 & $(\mathrm{GA})_{5} \mathrm{~A}(\mathrm{AG})_{30}$ & $\begin{array}{l}\text { GAACTCAAGAAGAATAAGGATG } \\
\text { TTGGTCGGCTAAGGATAC }\end{array}$ & 51 & 7 & $210-263$ & 0.4074 & 0.8379 & 0.0001 & 0.7980 \\
\hline
\end{tabular}




\begin{tabular}{|c|c|c|c|c|c|c|c|c|c|c|}
\hline Locus & $\begin{array}{l}\text { Accession } \\
\text { No. }\end{array}$ & Repeat motif & Primer sequence $\left(5^{\prime}-3^{\prime}\right)$ & $\begin{array}{c}\mathrm{Ta} \\
\left({ }^{\circ} \mathrm{C}\right)\end{array}$ & $N_{\mathrm{A}}$ & $\begin{array}{l}\text { Size range } \\
\text { (bp) }\end{array}$ & $H_{\mathrm{o}}$ & $H_{\mathrm{E}}$ & $P$ & PIC \\
\hline EC505 & JQ319405 & $(\mathrm{GA})_{31} . .(\mathrm{AG})_{23}$ & $\begin{array}{l}\text { CGAAGCAAACGAGAAGAG } \\
\text { ACAAGAATTGACGAGGGA }\end{array}$ & 56 & 8 & $260-312$ & 0.3478 & 0.8425 & $0.0000^{*}$ & 0.8011 \\
\hline EC41 & JQ319406 & $(\mathrm{TC})_{27}$ & $\begin{array}{l}\text { ATGACTCTTGGACGATTT } \\
\text { GCATTTATGGCTTTGGTAT }\end{array}$ & 54 & 10 & $294-336$ & 0.5000 & 0.8513 & 0.0185 & 0.8159 \\
\hline EC35 & JQ319407 & $(\mathrm{GA})_{24}$ & $\begin{array}{l}\text { CCTCAGTATGCCGTGTAG } \\
\text { CGAGCGGATGTAATAAAC }\end{array}$ & 54 & 7 & $330-387$ & 0.5185 & 0.8302 & 0.0076 & 0.7897 \\
\hline EC72 & JQ319408 & $(\mathrm{GA})_{26}$ & $\begin{array}{l}\text { GTCGGGAGAAGACAGGGAG } \\
\text { TGAAGAGGCGAGGTTGGT }\end{array}$ & 58 & 7 & $392-437$ & 0.5185 & 0.8204 & 0.0053 & 0.7776 \\
\hline $\mathrm{EC} 21$ & JQ319409 & $(\mathrm{GA})_{29}$ & $\begin{array}{l}\text { ACGTGCTTCTGGCAATCC } \\
\text { CTCGCCTTCATCCTCCTT }\end{array}$ & 58 & 10 & $258-312$ & 0.5000 & 0.8821 & $0.0000^{*}$ & 0.8487 \\
\hline EC1204 & JN408482 & $(\mathrm{CT})_{27}$ & $\begin{array}{l}\text { GAGTAGATAAAGAACACTGTGA } \\
\text { CTATTGAACAGGTTGACATAAG }\end{array}$ & 51 & 11 & $351-402$ & 0.6552 & 0.9026 & 0.0154 & 0.8763 \\
\hline EC1217 & JN408483 & $\begin{array}{l}(\mathrm{CT})_{27} . \\
(\mathrm{ACAT})_{6} . . \mathrm{A}_{18}\end{array}$ & $\begin{array}{l}\text { CTATTGAACAGGTTGACATAAG } \\
\text { TCCTCCAACAAAACCCTCAAAC }\end{array}$ & 62 & 8 & $352-390$ & 0.8000 & 0.8633 & 0.1022 & 0.8305 \\
\hline EC1218 & JQ319410 & $(\mathrm{TC})_{20}$ & $\begin{array}{l}\text { AAAGGAGTTATTCTCACCAAGG } \\
\text { ATATTGCTCTAATGCACCCAGG }\end{array}$ & 60 & 9 & $126-179$ & 0.6000 & 0.8506 & 0.0075 & 0.8142 \\
\hline EC38 & JQ319411 & $(\mathrm{GA})_{19} \mathrm{GG}(\mathrm{GA})_{12}$ & $\begin{array}{l}\text { GCGAATAGATTGGAAGAC } \\
\text { CAAATAAGAATTATGGGTGT }\end{array}$ & 51 & 10 & $392-437$ & 0.7083 & 0.8528 & 0.3060 & 0.8168 \\
\hline EC58 & JQ319412 & $(\mathrm{CT})_{32}$ & $\begin{array}{l}\text { CACCGCTTCTGGTATCTT } \\
\text { GTTGATAATAATGCCGAGA }\end{array}$ & 53 & 6 & $331-418$ & 0.1667 & 0.7934 & $0.0000^{*}$ & 0.7408 \\
\hline EC1222 & JQ319413 & $(\mathrm{AG})_{22}$ & 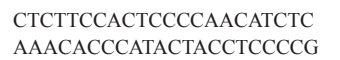 & 58 & 12 & $104-123$ & 0.5556 & 0.8560 & 0.0040 & 0.8242 \\
\hline EC620 & JN408484 & $(\mathrm{TC})_{9} \mathrm{G}(\mathrm{CT})_{29}$ & $\begin{array}{l}\text { CGATTCATCCGATACCAC } \\
\text { TTTCTGTCAATGCGTCCT }\end{array}$ & 62 & 8 & $189-225$ & 0.5217 & 0.8599 & 0.0268 & 0.8212 \\
\hline EC56 & JQ319414 & $(\mathrm{CT})_{24}$ & $\begin{array}{l}\text { CACTTCCACGCTGGTAAA } \\
\text { GACTGCGACTGGGTAATG }\end{array}$ & 62 & 2 & $154-167$ & 0.2593 & 0.2299 & 0.4773 & 0.2002 \\
\hline EC4 & JQ319415 & $(\mathrm{CT})_{31}$ & $\begin{array}{l}\text { CACCGCTTCTGGTATCTT } \\
\text { GTTGATAATAATGCCGAGA }\end{array}$ & 53 & 14 & $342-419$ & 0.3636 & 0.9228 & $0.0000^{*}$ & 0.8939 \\
\hline EC93 & JQ319416 & $(\mathrm{GA})_{14}$ & $\begin{array}{l}\text { AGGGGTGGGGTAAAAGCAAATA } \\
\text { CCGATGTTCTGGCTGTTCTCTA }\end{array}$ & 53 & 9 & $259-296$ & 0.2105 & 0.8734 & 0.0008 & 0.8341 \\
\hline EC1202 & JQ319417 & $(\mathrm{CT})_{30}$ & $\begin{array}{l}\text { TACTCAAATGGCAACACTCT } \\
\text { AGATAGAGGTAAAGTGAGCA }\end{array}$ & 56 & 8 & $320-413$ & 0.3478 & 0.8222 & $0.0000^{*}$ & 0.7792 \\
\hline EC506 & JN408487 & $(\mathrm{GA})_{23}$ & $\begin{array}{l}\text { TTTAGTTGGCGTCTTGGT } \\
\text { AGAACGAACAATGGAGGA }\end{array}$ & 56 & 10 & $316-348$ & 0.5200 & 0.8735 & $0.0000^{*}$ & 0.8411 \\
\hline EC1 & JQ319418 & $(\mathrm{GA})_{28} . .(\mathrm{AG})_{26}$ & $\begin{array}{l}\text { TGTATTGGAGGAGAAGCAGAAG } \\
\text { AGACAAGTCGGTGATGGTTTAG }\end{array}$ & 62 & 7 & $441-452$ & 0.4545 & 0.8312 & 0.0165 & 0.7649 \\
\hline EC92 & JQ319419 & $(\mathrm{CA})_{6}(\mathrm{AG})_{32}$ & $\begin{array}{l}\text { ATTCACAACCTAACCAGGAAAT } \\
\text { TTCTCAAAACGATTGTAAACCA }\end{array}$ & 56 & 9 & $79-126$ & 0.3684 & 0.8578 & $0.0000 *$ & 0.8167 \\
\hline EC503 & JQ319420 & $(\mathrm{TC})_{33}$ & $\begin{array}{l}\text { TACTTTCGCTCGCTAACA } \\
\text { TCCCGTTTCAAATTCTTGT }\end{array}$ & 54 & 7 & $224-263$ & 0.2500 & 0.7903 & $0.0000^{*}$ & 0.7347 \\
\hline EC619 & JQ319421 & $(\mathrm{CT})_{26}$ & $\begin{array}{l}\text { TGGTCATATTAAGAAAAGGTTG } \\
\text { ATGTCTGCGTTCCAATCC }\end{array}$ & 51 & 7 & $184-222$ & 0.2500 & 0.8659 & $0.0000^{*}$ & 0.8074 \\
\hline EC10 & JQ319422 & $(\mathrm{AG})_{23} \mathrm{AA}(\mathrm{AG})_{5}$ & $\begin{array}{l}\text { ACTTGAGACTGTTAGTGGAAAA } \\
\text { TATTGCGTGCTGGTTCTC }\end{array}$ & 53 & 10 & $444-521$ & 0.1000 & 0.8872 & $0.0000^{*}$ & 0.8516 \\
\hline EC1029 & JN408491 & $(\mathrm{GA})_{23} \mathrm{G}_{9}$ & $\begin{array}{l}\text { GAGATGGGTGAAGCAGAG } \\
\text { TACGTCGTAATGTGATGTATGT }\end{array}$ & 58 & 6 & $109-123$ & 0.4348 & 0.8048 & $0.0000^{*}$ & 0.7549 \\
\hline
\end{tabular}

Ta = annealing temperature; $N_{\mathrm{A}}=$ number of alleles; $H_{\mathrm{O}}=$ observed heterozygosity; $H_{\mathrm{E}}=$ expected heterozygosity; $\mathrm{P}=$ probability of being in Hardy-Weinberg equilibrium; PIC = polymorphism information content; *significant departure from expected Hardy-Weinberg equilibrium conditions after correction for multiple tests.

\section{ACKNOWLEDGMENTS}

Research supported by the Earmarked Fund for National "863" Project of China (\#2012AA10A409), the Modern Agro-Industry Technology Research System (\#CARS-47), the Special Fund for Agro-Scientific Research in the Public Interest (\#201103034), and in part by the Special Basic R\&D Fund of National Scientific Research Institutes with the Public Interest China (\#20603022012021). 


\section{REFERENCES}

Botstein D, White RL, Skolnick M and Davis RW (1980). Construction of a genetic linkage map in man using restriction fragment length polymorphisms. Am. J. Hum. Genet. 32: 314-331.

Li MY (1994). An approach to the reproduction and growth of shrimp Exopelaemon carinicauda cultured in earthen ponds with reference to its maximum sustaining yield on catch rotation. J. Fish. China 18: 85-92.

Liu P, Kong J, Shi T, Zhuang ZM, et al. (2000). RAPD analysis of wild stock of penaeid shrimp (Penaeus chinensis) in the China's coastal waters of Huanghai and Bohai Seas. Acta Oceanol. Sin. 22: 89-93.

Pemberton JM, Slate J, Bancroft DR and Barrett JA (1995). Nonamplifying alleles at microsatellite loci: a caution for parentage and population studies. Mol. Ecol. 4: 249-252.

Rousset F (2008). Genepop'007: a complete re-implementation of the genepop software for Windows and Linux. Mol. Ecol. Resour. 8: 103-106.

Shi DQ and Ye JS (2007). The biological characteristic and cultural technique of Exopalamon carinicauda holthuis. Fish Econ. Res. 5: 40-42.

Song CN, Li J, Liu P, Chen P, et al. (2010). Microsatellite-enriched library construction and polymorphic microsatellite marker isolation in Charybdis japonica. J. Fish. China 35: 35-42.

Wang XQ and Cao M (2010). Effects of low salinity and low temperature on survival, growth, and energy budget of juvenile Exopalaemon carinicauda. J. Hydroecol. 3: 66-71.

Xu WJ, Xie JJ, Shi H and Li C (2010). Hematodinium infections in cultured ridgetail white prawns, Exopalaemon carinicauda, in eastern China. Aquaculture 300: 25-31.

Yeh FC, Yang R, Boyle TJ and Ye Z (1999). POPGENE 32: Microsoft Windows-Based Freeware for Population Genetic Analysis. Molecular Biology and Biotechnology Centre. University of Alberta, Edmonton. 